\title{
Kernos
}

Revue internationale et pluridisciplinaire de religion grecque antique

21 | 2008

Varia

\section{De la philologie selon Jean Rudhardt et de la compréhension d'autrui}

André Hurst

\section{(2) OpenEdition \\ Journals}

Édition électronique

URL : https://journals.openedition.org/kernos/1609

DOI : 10.4000/kernos.1609

ISSN : 2034-7871

\section{Éditeur}

Centre international d'étude de la religion grecque antique

\section{Édition imprimée}

Date de publication : 1 janvier 2008

Pagination : 131-137

ISSN : 0776-3824

\section{Référence électronique}

André Hurst, « De la philologie selon Jean Rudhardt et de la compréhension d'autrui », Kernos [En ligne], 21 | 2008, mis en ligne le 01 octobre 2011, consulté le 24 août 2022. URL : http:// journals.openedition.org/kernos/1609; DOI : https://doi.org/10.4000/kernos.1609 


\section{De la philologie selon Jean Rudhardt et de la compréhension d'autrui}

Tout lecteur de Jean Rudhardt aura perçu la nuance très visible de parodie affectueuse contenue dans le titre de ces quelques considérations.

On voudra bien tolérer, voire pardonner cette forme de l'hommage.

On voudra bien permettre, également, que ma contribution à ce colloque se présente ouvertement comme celle d'un ancien étudiant de Jean Rudhardt, ancien étudiant qui eut la chance de devenir par le jeu des circonstances un collaborateur proche dans le cadre de l'édition du «Codex des Visions » de la Bodmeriana.

Je m’éloignerai par conséquent jusqu'à un certain point de ce qui constitue la conformité ordinaire avec l'horizon d'attente d'un texte "scientifique », et serai conduit à évoquer des souvenirs personnels.

Mon premier souvenir de rencontre avec Jean Rudhardt se situe à l'époque où, étudiant à la Faculté des Lettres de l'Université de Genève, j'étais astreint à des exercices de thème grec en vue d'un examen de licence. C'est Jean Rudhardt qui dirigeait ces exercices et nous eûmes la surprise de l'entendre affirmer d'entrée de jeu que le thème grec était, je cite, « un exercice passionnant». Cette idée, inutile de le préciser, ne nous aurait pas effleurés un seul instant à cette époque. Jean Rudhardt nous était connu par le titre de sa thèse de doctorat, dont la minutieuse précision et l'étendue nous impressionnaient, et je ne parle encore que du seul titre! -, avant même que nous eussions eu l'occasion d'en examiner le contenu. Un coup d'œil jeté à cette thèse nous persuadait d'ailleurs qu'une même idée se trouvait sous-jacente aussi bien au thème grec que nous pratiquions sous la direction de son auteur qu'à l'analyse serrée du vocabulaire grec relatif aux faits religieux présentée dans ce livre: il existe des mots intraduisibles, il faut dans toute la mesure du possible devenir celui qui les a écrits ou prononcés pour en saisir la portée conceptuelle et, surtout, pour percevoir la différence qui existe entre le monde de celui qui parle et le nôtre. Jean Rudhardt a donné forme systématique aux fondements de son enseignement du thème grec dans une étude remarquable publiée en 1964 dans 
les « Cahiers Ferdinand de Saussure » $(21,1964)^{1}$. J'en citerai une formule qui illustre ce qu'était pour nous son enseignement : « ... on découvrirait même, en étudiant les uns après les autres tous les mots du dictionnaire, que les signifiés grecs et français ne se recouvrent presque jamais exactement. » Plus récemment encore, il écrivait: "Il est difficile d'entrer dans la subjectivité d'autrui; ce l'est particulièrement, quand plus de deux mille ans vous séparent des hommes que

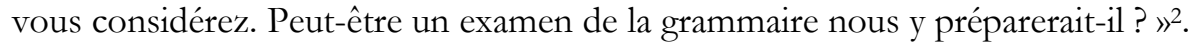

Or, ce qui ne manquait pas d'impressionner, c'est que cette quête du sens contenu dans le message d'autrui n'avait rien d'une passion grammaticale, ou alors, si l'on veut, c'était la vraie passion grammaticale poussée à son point le plus noble. Nous étions entrainés dans une recherche de la signification qui participait d'un projet ambitieux de connaissance de soi au travers d'une exploration aussi minutieuse que possible des différences d'avec autrui. Dans ce sens, l'examen de la possibilité ou de l'impossibilité de faire passer en grec un texte français se situait sur le même plan que la recherche ardente de ce qui pouvait justifier, sur un papyrus documentaire, le payement de telle taxe à l'époque impériale romaine, ou que la recherche du sens à déchiffrer dans un geste rituel athénien attesté à l'époque classique. On se trouvait en quête d'un fonctionnement de la pensée. C'est ainsi que Jean Rudhardt écrit, dans l'essai déjà mentionné : "Il nous faut donc, dans la mesure où nous avons correctement posé le problème, reconnaitre que l'actualisation ne résulte pas des relations que la langue, par la syntaxe, établit entre les idées signifiées par les mots; nous devons en d'autres termes, bien que l'actualité du mot appartienne à la langue, admettre que le processus de son actualisation n'est pas un fait linguistique, mais un acte original et dans une certaine mesure autonome de la pensée » $\aleph^{3}$.

Cet acte original et dans une certaine mesure autonome de la pensée sera traqué par Jean Rudhardt tout au long des études qu'il va consacrer à la religion grecque, aux récits mythologiques, aux rites.

Une occasion de la traquer dans un domaine nouveau fut du même coup pour moi l'occasion de collaborer de près avec mon ancien maître: la Fondation Bodmer nous confia l'édition du dernier codex encore inédit du lot que le collectionneur Martin Bodmer avait pu acheter dans les années 1950 au Caire, un lot de papyrus, on le sait, qui constituait déjà une collection dans l'antiquité. Ce codex de papyrus contenait divers textes, dont un seul était déjà connu : il s'agissait d'une partie du «Pasteur» d'Hermas (les trois premières

${ }^{1}$ Nous citerons d'après la publication de ses articles dans Cabiers Vilfredo Pareto, Revue européenne des sciences sociales 19 (1981), n ${ }^{\circ}$. L'étude en question, "Réflexions philosophiques à l'occasion d'un exercice de traduction ", s'y trouve reprise aux p. 33-57.

2 Jean RUDHARDT, «Considérations rapides sur la conscience de soi dans la Grèce antique », SMSR 62 (1996), p. 471.

${ }^{3}$ RUDHARDT, o.c. (n. 1), p. 41. 
«Visions » de ce texte). Tout le reste du document était inédit. Comme l'un des inédits de ce codex, celui qui faisait suite directement au texte connu d'Hermas, portait clairement pour titre «Vision de Dorothéos », le codex, à la suggestion de Rodolphe Kasser, avait été nommé "Codex des visions », un titre dont on s'aperçut qu'il ne recouvrait qu'une partie du contenu de ce cahier ${ }^{4}$, mais qu'il fut décidé de conserver pour éviter de créer la confusion.

Il convient de rappeler que «nous» était au départ un collectif de trois personnes: Jean Rudhardt, Olivier Reverdin, moi-même. De fait, Olivier Reverdin reconnut très vite que ses lourdes obligations politiques, de même que sa charge dans le domaine de la politique de la science, ne lui permettaient pas de consacrer à ce travail tout le temps et toute l'attention qu'il aurait voulu y mettre. C'est la raison principale pour laquelle, après la publication de la "Vision de Dorothéos ", Jean Rudhardt et moi-même nous retrouvâmes à deux pour ce qui constituait l'essentiel du codex, à savoir les poèmes sur des sujets divers, dont les liens avec la "Vision de Dorothéos » et le «Pasteur» allaient ouvrir des perspectives sur les propriétaires antiques du lot de papyrus acheté naguère par Martin Bodmer.

Je citerai deux épisodes de cette collaboration, deux épisodes qui sont de nature à expliquer quel type d'« autrui » fut Jean Rudhardt dans cette aventure intellectuelle, et comment deux de ses réactions extrêmes peuvent ouvrir à la compréhension de sa pensée et de sa personnalité.

Le premier épisode concerne notre travail sur le poème "Adresse à Abraham », premier texte à faire suite à la «Vision de Dorothéos ». On relèvera que ce poème n'est pas une vision, et qu'à la suite des textes du «Pasteur» et de la "Vision de Dorothéos » on entre par conséquent dans un segment nouveau du codex. Ce poème raconte de manière originale le sacrifice d'Isaac, et nous n'allons pas y revenir, sinon pour rappeler que les éditeurs de ce texte se trouvaient devant une circonstance qui constituait tout à la fois une aide et une difficulté : le poème est acrostichique, c'est-à-dire que du vers 6 au vers 27, les premières lettres de chaque vers produisent la séquence de l'alphabet. L'auteur (ou le copiste) le souligne d'ailleurs en indiquant, "extra versum» après le

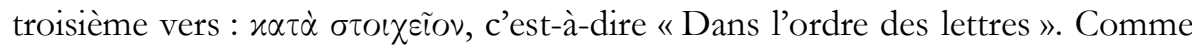
un certain nombre de vers sont mutilés en leur début, l'éditeur dispose d'un guide qui constitue également un indiscutable carcan : on sait pertinemment par quelle lettre le vers en question doit débuter.

Sur un point, parmi d'autres, la version des faits présentée par ce poème diffère de la version connue de l'Ancien Testament: Isaac sait quel sort lui est

\footnotetext{
${ }^{4}$ Les deux descriptions du codex par Rodolphe Kasser montrent tout à la fois la familiarité de ce savant avec un codex qu'il avait eu entre les mains parmi les tout premiers, et son extrême scrupule à corriger ce qui était apparu, après la première publication de sa description en appendice à la «Vision de Dorothéos », comme une erreur dans la disposition des bifolios du codex (ainsi le codex débutait par l'extrait d'Hermas, au lieu de se conclure par lui).
} 
réservé, il s'en réjouit et exhorte ses parents à le sacrifier (en conformité avec une tradition juive du courage d'Isaac). Voici quel langage lui fait tenir l'auteur du poème :

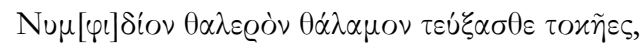

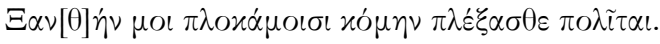

Abr. 16-17

Construisez pour moi la chambre nuptiale convenant à ma jeunesse, vous mes parents,

Tressez-moi une flambante chevelure, vous mes concitoyens, etc.

Sans nous en apercevoir, Jean Rudhardt et moi-même n'étions pas d'accord sur la manière de comprendre ces deux vers. L'élément qui fit apparaître le désaccord fut ma suggestion de rapprocher le mot $\xi \alpha \nu \theta \eta \dot{\nu}$ d'un passage de Bacchylide où il est question de l'exécution de Crésus sur un bûcher. Bacchylide raconte qu'au moment où l'on alluma le bûcher, Zeus, en récompense de la piété de Crésus, fit pleuvoir et éteignit ainsi la « blonde flamme ».

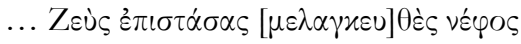

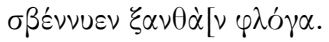

Zeus fit planer un noir nuage

Bacch. 3, 55-56

Il éteignit la blonde flamme.

Lors de nos rencontres, Jean Rudhardt résistait à ce rapprochement, qui m'était dicté par la présence dans chacun des deux textes non seulement du

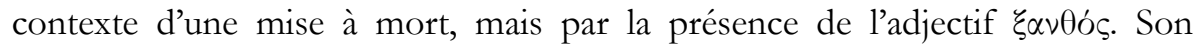
argumentation portait sur la différence des contextes, que j'interprétais naïvement comme la différence des conditions de production des œuvres ainsi rapprochées. Au bout de quelques séances, je m’aperçus que je m’étais mal fait comprendre: pour moi, la «blonde chevelure » évoquée par Isaac n’était pas celle qu'il portait sur la tête, mais ces mots désignaient métaphoriquement les flammes du bûcher, comme chez Bacchylide, avec la même nuance de couleur pour la flamme. "Alors, me dit à peu près Jean Rudhardt, tu veux dire que ce vers adressé aux concitoyens leur demande de mettre le feu au bûcher?». «Oui : les parents construisent l'autel du sacrifice (c'est la « chambre nuptiale ») et les autres mettent le feu, c'est le sens de cette « chevelure blonde » qu'il faut « tresser».

Je vis Jean Rudhardt réfléchir un bref instant, puis il me dit: "Alors on oublie tout ce que j'ai dit, c'est évident et je ne vois plus d'autre manière possible de comprendre ces mots. »

Je fus abasourdi par l'instantanéité de cette prise de position nouvelle. Elle témoignait d'abord de beaucoup d'indulgence à mon égard : je m'étais mal fait comprendre, mais cela ne lui importait en rien. Elle témoignait, en outre, d'une agilité vraiment scientifique. Lorsque les arguments convainquent, on oublie ce 
qu'on avait construit soi-même, car l'évidence prend le dessus. Je reçus à cette occasion une bonne leçon: Jean Rudhardt venait de s'investir avec ardeur, comme il le faisait toujours, dans la défense de son point de vue. Mais cette ardeur ne l'empêchait en rien de renoncer à ce point de vue lorsque lui apparaissait ce qu'il considérait désormais comme la bonne solution.

Voilà pour le premier des deux épisodes.

Le deuxième est à l'opposé : il s'agit d'un point sur lequel, au contraire de ce qui s'est passé avec la chevelure d'Isaac, nous n'avons jamais pu tomber d'accord.

L'« Adresse à Abraham », une nouvelle fois, fut le point de départ. Voici ce qui est dit au tout début du texte :

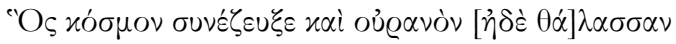

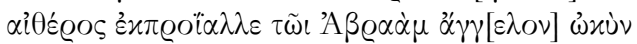

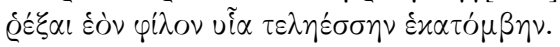

Celui qui créa le monde, avec le ciel et la mer,

Envoya du haut de l'éther un ange rapide vers Abraham,

Avec l'ordre d'immoler son propre fils en sacrifice solennel.

Nous nous posâmes évidemment la question de savoir si cette présentation de l'univers créé par Dieu reflétait ou non une physique reposant sur la théorie des quatre éléments. Ma réponse était clairement oui, celle de Jean Rudhardt était clairement : on n'a pas les moyens de le savoir.

À mes yeux, et c'est sans doute la solution la plus naïve, la mention de l'« éther » indiquait allusivement une telle vision du monde. Dans la conception biblique, Dieu n'envoie pas de messagers d'un lieu qui ne serait pas son propre lieu de résidence, et le «feu» contenu dans la notion d'«éther» n'en fait pas partie. Si, par conséquent, l'ange était explicitement montré comme envoyé de la partie «flambante» du monde, c'est que ce feu complétait le schéma des quatre éléments, les trois autres étant l'air (= le ciel, ở @avóv), l'eau (= la mer, $\theta \dot{\alpha} \lambda \alpha \sigma \sigma \alpha \nu)$ et la terre (= le monde, $x o ́ \sigma \mu o \nu)$. Il y a certes des faiblesses dans ces rapprochements : le «monde » ne serait-il pas plutôt l'ensemble de la création ? Oui, mais l'expression utilisée (littéralement : «joignit ensemble le monde, le

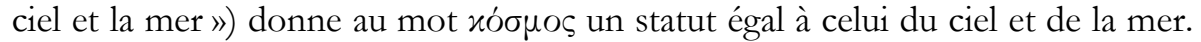
Il n'est donc pas ce qui inclut tout, il est bien plutôt un élément comme les autres.

Je vous épargne de longues discussions, qui invariablement se terminaient par l'expression du doute et du scepticisme de la part de Jean Rudhardt, même si je faisais référence au fait que le « Pasteur» d'Hermas, par lequel s'ouvrait le "Codex des Visions », mentionnait explicitement la théorie physique des quatre éléments. Ajoutons que cela n’impliquait aucune originalité, ni de ma part, bien sûr, ni de la part des auteurs antiques qui auraient recouru à cette conception du monde : c'était le filtre d'explication physique de l'univers le plus répandu, et 
cela au moins depuis Empédocle d'Agrigente. Or, et de surcroit, un mot rare

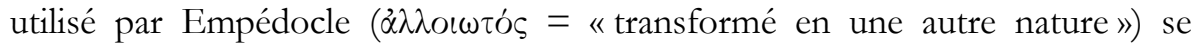
trouvait utilisé dans la «Vision de Dorothéos » (cf. VD. 273) ...

Rien n'y fit: Jean Rudhardt n'était pas convaincu. Comment allions-nous traiter le commentaire de ce passage dans un livre que nous devions signer ensemble? On trouvera la réponse à cette question à la page 50 de notre édition des «Poèmes divers $»^{5}$. La note commence par le point important qui consistait à éviter les nombreuses confusions que d'aucuns avaient voulu entretenir entre les textes du «Codex des visions » et le monde des gnostiques. Le verbe $\sigma \cup v \varepsilon ́ \zeta \varepsilon \cup \xi \varepsilon$ leur offrait une possibilité, compte tenu du groupement par

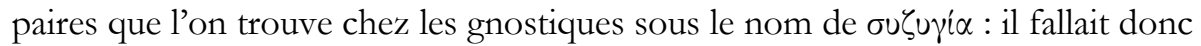
souligner que l'on se trouve ici dans un tout autre contexte, qui est celui de la création du monde matériel.

Puis, c'est la suite de cette note, on se pose la question du schéma des quatre éléments. Les arguments en faveur de cette hypothèse une fois énoncés, vient un paragraphe débutant par «Observons toutefois que ... », lequel introduit toute la réticence de Jean Rudhardt, réticence désormais fondée sur le fait qu'il ne peut admettre sans autres que le mot «éther» soit ici le représentant de l'élément «feu». Il le voit plus immédiatement comme l'expression du lieu habité par le dieu créateur, donc l'équivalent du « ciel ».

La rédaction de cette note résulte, on s'en doute, d'échanges qui s'étendirent sur plusieurs mois à propos du passage en question. Le résultat peut être ainsi résumé : les opinions divergentes sont exprimées aussi complètement que chacun de nous pouvait le faire, et elles coexistent sans polémique, dans le respect non seulement l'un de l'autre pour ce qui est des éditeurs, mais surtout dans le respect du lecteur: à lui de choisir en connaissance de tous les arguments que nous avons pu avancer, ou, mieux encore, d'en trouver de meilleurs.

Les deux épisodes choisis illustrent à mes yeux un comportement constant chez Jean Rudhardt: la compréhension d'autrui se fonde sur une valeur

\footnotetext{
${ }^{5}$ Le Codex des Visions est publié en trois volumes, indiqués ici dans l'ordre qui correspond à celui des textes dans le codex :

Papyrus Bodmer XXXVIII. Erma, Il Pastore (Ia-IIIa visione), Edito con introduzione e commentario critico da Antonio Carlini (con la collaborazione di Luigi Giaccone). Appendice: Nouvelle description du Codex des Visions par Rodolphe Kasser, avec la collaboration de Guglielmo Cavallo et Joseph van Haelst, Cologny/Genève, 1991.

Papyrus Bodmer XXIX. Vision de Dorothéos, Édité avec une introduction, une traduction et des notes par André Hurst, Olivier Reverdin, Jean Rudhardt. En appendice : Description et datation du Codex des Visions par Rodolphe Kasser et Guglielmo Cavallo (cette description du codex est désormais remplacée par celle de 1991), Cologny/Genève, 1984.

Papyri Bodmer XXX-XXXVII. "Codex des Visions» Poèmes divers, Édités avec une introduction générale, des traductions et des notes par André Hurst et Jean Rudhardt, München, 1999.
} 
sous-jacente qui n'est pas exprimée en tant que telle, et qui n'est autre que le respect d'autrui.

«Compréhension d'autrui », dans cette perspective, n'est pas une simple formule. Comme on le constate, j'ai pu en faire l'expérience à plus d'un titre. Nous avons ensemble tenté de comprendre ce qu'étaient ces «autres » souvent bizarres qui se cachaient derrière des textes dans lesquels ils prétendaient cependant révéler les valeurs les plus profondes auxquelles ils étaient attachés (je songe surtout, mais pas exclusivement, au poème intitulé «Adresse aux Justes »). De surcroît, j’ai pu faire l'expérience privilégiée de la compréhension d'autrui dans le cadre de notre collaboration : compréhension signifie ici tout à la fois attention portée à l'argumentation de l'autre (le cas de la chevelure d'Isaac) et délimitation claire de son point de vue devant les suggestions d'autrui (l'épisode des quatre éléments). Il ne s'agit donc pas, dans la compréhension d'autrui, et même si l'on préconise comme Jean Rudhardt de se « faire Grec», de se fondre dans l'altérité, comme tel personnage loufoque du «Cavaliere inesistente» d'Italo Calvino qui, à chaque rencontre, devient littéralement son vis-à-vis par une métamorphose totale de son comportement. Le passage par l'indispensable compréhension d'autrui doit finalement déboucher sur un enrichissement de la connaissance de soi et non sur une perte de soi.

Dernière touche philologique, pour compléter ce qui vient d'être évoqué tout en apportant un élément qui a son importance dans ce qui fut la quête constante de Jean Rudhardt : il est évident que, dans le titre de ces pages, «De la philologie selon Jean Rudhardt et de la compréhension d'autrui », le mot « et » doit être pris dans son sens "épexégétique », un usage bien connu des grammairiens grecs; il s'agit du cas où le mot « et » n'introduit pas d'élément supplémentaire, mais bien une explication de ce qui précède, un peu comme si l'on disait « c'est-à-dire », ou " à savoir ». On l'aura compris en effet : le ressort qui anime la recherche de Jean Rudhardt est celui-là : pouvoir entrer sur le territoire conceptuel de l'autre, quitte à manifester par la suite des points de désaccord. Il était évident que Jean Rudhardt se donnait les moyens de juger, mais pas avant d'avoir exploré le «monde d'autrui », mots, textes, rituels, au plus près de sa conscience. Cependant, à vouloir se «faire Grec », il lui est souvent arrivé de le devenir vraiment. Ainsi, lorsqu'il décrivait la structure des récits théogoniques des Grecs et les relations qu'ils tissaient entre les origines et le monde présent, les mots «aujourd'hui encore» étaient à comprendre en général dans sa bouche comme signifiant «au siècle de Périclès »... Mais la compréhension d'autrui vaudrait-elle qu'on s'y aventure si l'on en sortait systématiquement indemne?

André HURST

Université de Genève

Département des Sciences de l'Antiquité

CH - 1211 Genève 4

Courriel: andre.burst@lettres.unige.ch 